\title{
Quantitative Ventilation/Perfusion Tomography: the Foremost Technique for Pulmonary Embolism Diagnosis
}

\author{
Marika Bajc and Jonas Jögi \\ Department of Clinical Physiology, \\ Lund University and Skåne University Hospital, Lund
}

Sweden

\section{Introduction}

The value of perfusion scintigraphy in the detection of pulmonary embolism (PE) was demonstrated as early as 1964 by Wagner et al. PE causes perfusion defects that conform to the anatomical distribution of the pulmonary vascular bed. Perfusion defects in acute PE are therefore of sub-segmental, segmental or lobar character. Ventilation is normally preserved in these areas and the observed wedge shaped mismatch between ventilation and perfusion is typical for PE. Planar ventilation/perfusion scintigraphy (V/P scan) was until the 1990s the method of choice for studying patients with suspected PE. However, the large Prospective Investigation of Pulmonary Embolism Diagnosis (PIOPED I), showed a high number of non-diagnostic examinations $(65 \%)$ with $\mathrm{V} / \mathrm{P}$ scan and the probabilistic interpretation criteria were confusing to the clinicians (Gray et al., 1993; The PIOPED Investigators, 1990). Planar imaging has become obsolete, particularly when the issue is identification and quantification of focal or regional aberration of organ function.

The advantage of three dimensional tomography over planar imaging for PE detection had already been shown in 1983 in a study on dogs (Osborne et al., 1983). Furthermore, Magnussen et al. (1999) used a computerized model of PE to highlight the advantage of SPECT over planar imaging in the assessment of the size and location of perfusion defects. Using a dual head camera, Palmer et al. (2001), developed a fast and efficient method for ventilation/perfusion tomography (V/P SPECT ) for clinical practice with total acquisition time of only 20 minutes. Moreover, they developed an algorithm to calculate the quotient between ventilation and perfusion and to present it as $\mathrm{V} / \mathrm{P}_{\text {quotient }}$ images. This facilitated $\mathrm{PE}$ diagnosis and the quantification of PE extension, which led to the use of the term quantitative V/P SPECT. Using a porcine model, Bajc et al. validated V/P SPECT for diagnosis of PE and confirmed the superior value of tomography over planar imaging with excellent interobserver agreement of defects down to the sub-segmental level (Bajc et al., 2002b).

The objective of this chapter is to acquaint readers with the latest methodological approach of $\mathrm{V} / \mathrm{P}$ SPECT in the diagnosis of PE, in accordance with the new guidelines of the European Association of Nuclear Medicine (Bajc et al., 2009a, b). In this chapter we also discuss the value of V/P SPECT in the follow up after acute PE and in the diagnosis of other cardiopulmonary diseases. 
Efficient and effective diagnostics for PE and other diseases should meet the following basic requirements:

- Fast procedure and prompt availability of results

- Feasibility for all patients

- High diagnostic accuracy and few non-diagnostic reports

- Low radiation dose

- Utility for selection of treatment strategy

- $\quad$ Suitability for follow up and research

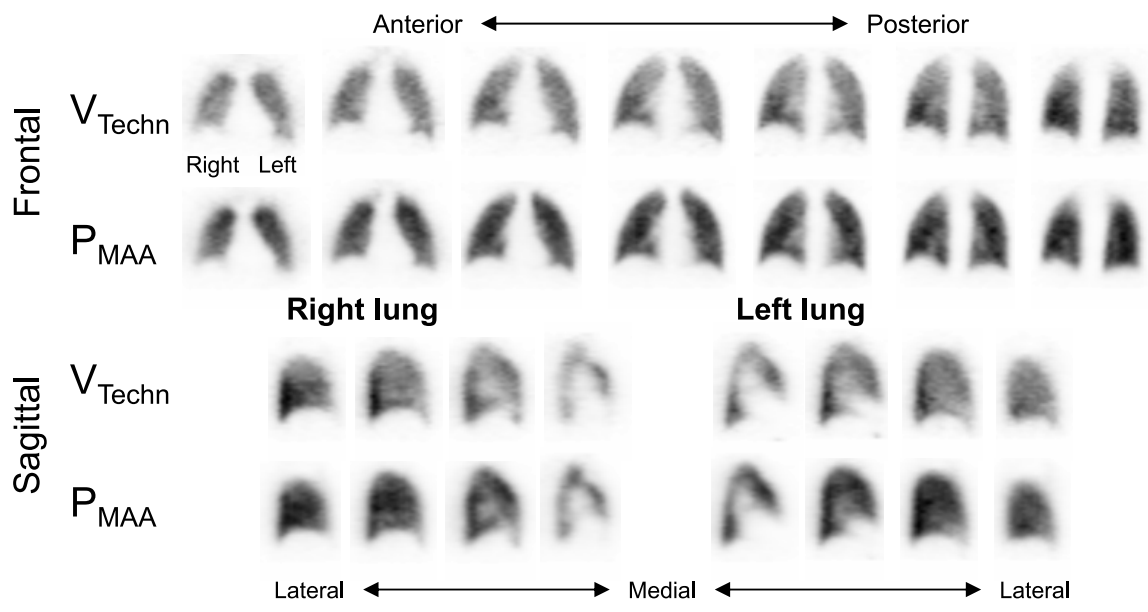

Fig. 1. V/P SPECT images of a patient with normal ventilation $(\mathrm{V})$ and perfusion $(\mathrm{P})$. Techn $=$ Technegas, MAA $=$ Macroaggregated albumin

\section{Ventilation and perfusion imaging}

In healthy patients, regional ventilation and perfusion match each other to optimize gas exchange (Fig 1). In diseased patients, changes in distribution of ventilation or perfusion or both are common. Vascular occlusive diseases, like PE, cause perfusion defects in conformity with pulmonary circulation while ventilation stays intact in these areas. This disconcordant ventilation/perfusion pattern, so called mismatch, provides the basis for PE diagnosis. Ventilation might be disturbed in acute PE due to bronchial constriction (Giuntini, 2001) but perfusion defects are usually observable in other areas (Fig 2a).

Ventilation commonly shows disturbances in lung diseases like pneumonia, tumours and obstructive diseases. Such perfusion patterns are essential to recognize as they provide additional specificity and significance to observed conditions.

\subsection{Ventilation}

For the ventilation study, gases may be used as they are distributed strictly according to regional ventilation. The gas that is used for V/P SPECT is metastable 81 -krypton $(81 \mathrm{mKr})$. Its short half life, $13 \mathrm{~s}$, implies that it disappears from the alveoli by decay at a much faster rate than by exhalation. Therefore, after a few minutes of breathing the test gas, the alveolar 


\section{A Initial examination}

\section{B Follow up after 3 days}

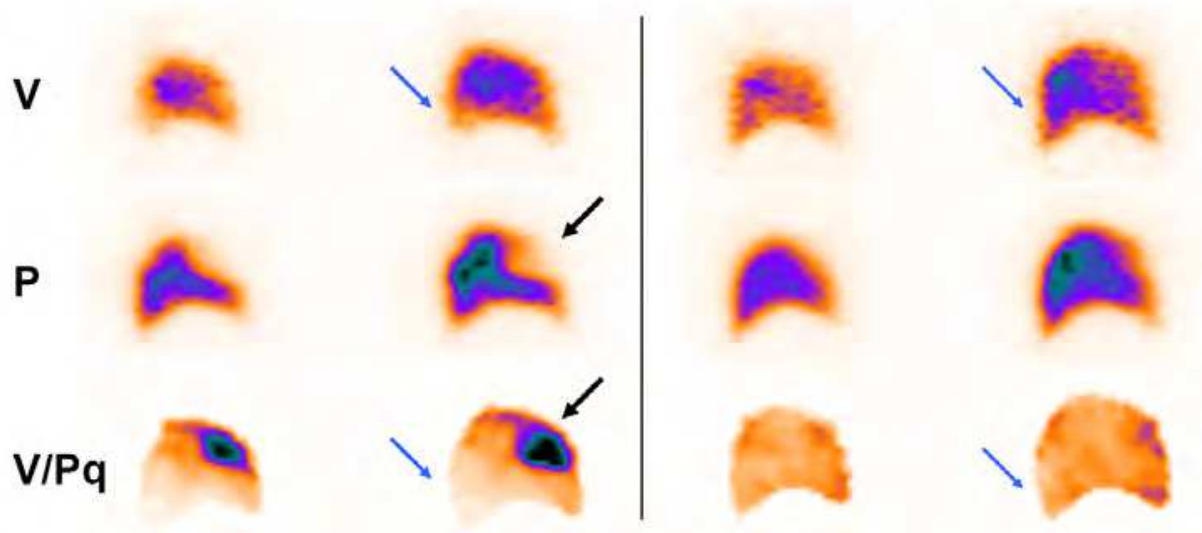

Fig. 2. A patient with acute PE. Sagittal ventilation $(\mathrm{V})$, perfusion $(\mathrm{P})$ and $\mathrm{V} / \mathrm{P}$ quotient $(\mathrm{V} / \mathrm{Pq})$ images of the right lung. A) At the initial examination, segmental perfusion defects are seen (arrow). The $\mathrm{V} / \mathrm{P}$ mismatch is clearly delineated on $\mathrm{V} / \mathrm{P}$ quotient images which improves visualization. Reduced ventilation is observed in posterior parts of the lung, where perfusion is preserved (blue arrows). B) Normalization of ventilation (blue arrows and perfusion is seen already after three days.

concentration will reflect alveolar ventilation. Ventilation is performed during continuous breathing of this gas. $81 \mathrm{mKr}$ has higher gamma energy than 99m-Technetium (99mTc) (191 compared to $140 \mathrm{keV}$ ) allowing simultaneous imaging of ventilation and perfusion. $81 \mathrm{mKr}$ is diluted from a rubidium generator that has a half life of $4.6 \mathrm{~h}$. Its availability is limited and it is too expensive for general use.

Routinely in clinical practice, inhalation of a radio-aerosol is used for ventilation scintigraphy. Aerosol particles are liquid or solid. The size of the particles is of critical importance. Particles larger than $2 \mu \mathrm{m}$ are deposited in large airways. Smaller particles are deposited by sedimentation and diffusion in small airways and alveoli. Particles smaller than $1 \mu \mathrm{m}$, are mainly deposited in alveoli by diffusion. Aerosol deposition is modified by flow pattern. High flow rates at forced breathing patterns and turbulent flow enhances particle deposition in airways and increases the likelihood of hot spots formation on ventilation images, particularly in Chronic Obstructive Pulmonary Disease (COPD).

Diethylenetriaminepentaacetic acid labeled with technetium, 99mTc-DTPA, is the most common agent used for ventilation scintigraphy. It is soluble in water and the size of the molecule is 492 Dalton. The average size of particles after nebulization is at best 1.3 to 1.8 $\mu \mathrm{m}$. Due to the water solubility, particle size tends to increase during inhalation and to agglutinate in cases of bronchial obstruction where there are turbulent flows; this leads to the creation of hot spots. Because of the water solubility, 99mTc-DTPA particles also diffuse through the alveolo-capillary membrane to the blood. In a healthy patient, clearance of 99mTc-DTPA occurs with a half life of about 70 minutes. Increased clearance, leading to a shorter half life is observed where there is alveolar inflammation for any reason, such as alveolitis of an allergic or toxic nature and even in smokers. Clearance of 99mTc-DTPA can for diagnostic purposes be measured at a routinely performed V/P SPECT. 
Technegas is a newer solid aerosol with extremely small carbon particles, 0.005-0.2 $\mu \mathrm{m}$, labeled with $99 \mathrm{mTc}$ which are generated in a high temperature furnace. The small particle size implies that they are distributed in the lungs almost like a gas and are deposited in alveoli by diffusion (James et al., 1992). Technegas provides images which are equivalent to those with $81 \mathrm{mKr}$. Technegas significantly reduces problems of central airway deposition and peripheral hotspots. Patients routinely admitted for V/P SPECT and a group of patients with known COPD were recently studied with both 99mTc-DTPA and Technegas showing superiority of the latter (Jögi et al., 2010). Unevenness of radiotracer deposition and degree of central deposition were significantly reduced with Technegas, particularly in the obstructive patients (Fig 3). In some patients, mismatched perfusion defects were only identified using Technegas because the significant peripheral unevenness of 99mTc-DTPA obscured mismatch. PE might have been overlooked in COPD patients using 99mTc-DTPA. In a few patients, $99 \mathrm{mTc}$-DTPA yielded images of very poor quality. Technegas is therefore recommended as the superior radio-aerosol, particularly in patients with obstructive lung disease. A further advantage of Technegas is that relatively few breaths are sufficient to achieve an adequate amount of activity in the lungs.
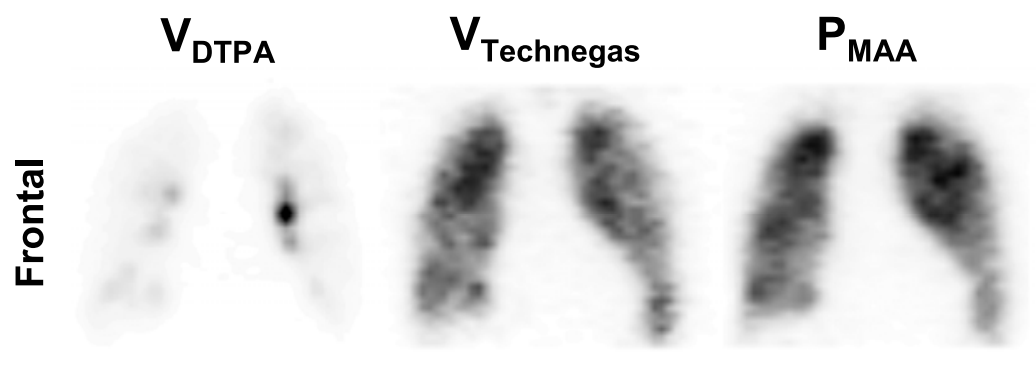

Fig. 3. Comparison between 99mTc-DTPA and Technegas ventilation studies in a patient with COPD.

\subsection{Perfusion}

Perfusion scintigraphy involves an intravenous injection of radio-labeled macroaggregates of albumin (MAA), sized 15-100 $\mu \mathrm{m}$, which cause microembolization of pulmonary capillaries and pre-capillary arterioles in amounts reflecting regional perfusion. At least 60000 particles are required to obtain a representative activity distribution (Heck \& Duley, 1974). Routinely, about 400000 particles are injected. As there are over 280 billion pulmonary capillaries and 300 million pre-capillary arterioles, only a very small fraction of the pulmonary bed will be obstructed. A preparation of 100000 200000 particles is recommended for patients with known pulmonary hypertension or after a single lung transplant. Degradation of MAA results in its elimination from the lung within a few hours.

\subsection{Acquisition}

To perform V/PSPECT takes only one hour from referral to report (Bajc et al., 2004; Palmer et al., 2001). The ventilation study starts with inhalation of 25-30 megabecquerel (MBq) Technegas, usually 2-3 breaths. Immediately after ventilation SPECT, a dose of 100-120 MBq 99mTc-MAA is given intravenously for perfusion imaging. 
During the examination, the supine patient carefully maintains the position between ventilation and perfusion acquisitions. Immobilization for only 20 minutes is well tolerated by nearly all patients. Examination in supine position is comfortable even for critically ill patients. It is also more convenient for the staff. It is noteworthy that V/P SPECT can be performed in all patients, since there is no contraindication related to age, radiation, contrast media or co-morbidity.

\subsection{Reconstruction and calculation of V/P quotient images}

Iterative OSEM is essential for SPECT reconstruction. Ventilation activity is subtracted from perfusion images. A valuable parameter in clinical SPECT is the Ventilation/Perfusion

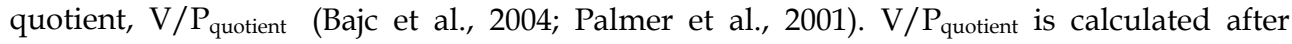
normalization of ventilation counts to perfusion counts. Hot spot removal is important, especially when $99 \mathrm{mTc}-\mathrm{DTPA}$ is used.

\subsection{Presentation of V/P SPECT}

$\mathrm{V} / \mathrm{P}$ SPECT images are usually presented in frontal, sagittal and transversal projections, available in any modern system. The slices must be accurately aligned so that ventilation and perfusion slices match each other for correct comparison. Therefore, it is crucial to achieve this acquisition in one session with maintained body position. This is also a prerequisite for the calculation of $\mathrm{V} / \mathrm{P}_{\text {quotient }}$ images, which greatly facilitates identification of ventilation/perfusion mismatches typical of $\mathrm{PE}$ as well as other patterns characteristic of other pulmonary diseases.

Volume rendered images, such as "Maximum Intensity Projection" are available with almost all SPECT systems, allowing rotating 3D views. This function is another valuable option, particularly for quantification and follow-up of PE patients.

\section{Interpretation}

According to the new European guidelines, the holistic interpretation of lung SPECT is recommended (Bajc et al., 2009b). The clinician can only benefit from reports, which clearly express the presence or absence of PE. This goal was not achieved with previous probabilistic reporting methods according to PIOPED or modified PIOPED. Large $\mathrm{V} / \mathrm{P}$ SPECT studies show that this is achievable if all patterns are considered, where these combine ventilation and perfusion. Conclusive reports were given in 97 to $99 \%$ of studies.

Holistic interpretation of V/P SPECT should be based upon: a) Clinical pre-test probability and $b$ ) the application of criteria for interpreting $\mathrm{V} / \mathrm{P}$ patterns to distinguish between patterns indicative of PE and other diseases.

\subsection{Criteria for acute PE according the european guidelines}

In accordance with the guidelines of the European Association of Nuclear Medicine (Bajc et al., 2009a, b), PE is reported if there is:

- $\mathrm{V} / \mathrm{P}$ mismatch of at least one segment or two sub-segments that conforms to the pulmonary vascular anatomy.

No PE is reported if there is:

- normal perfusion pattern conforming to the anatomic boundaries of the lungs,

- matched or reversed mismatch V/P defects of any size, shape or number in the absence of mismatch 
- mismatch that does not have a lobar, segmental or sub-segmental pattern Non-diagnostic for PE is reported if there are:

- $\quad$ Multiple V/P abnormalities not typical of specific diseases.

The fundamental point is that lobar, segmental or sub-segmental $\mathrm{V} / \mathrm{P}$ mismatch serves as the basis for confirming a diagnosis of PE among patients with suspected PE. Howarth et al. state that: "at least 0.5 of a segment of ventilation/perfusion mismatch is considered diagnostic of PE" (Howarth et al., 2006). This simplified criterion gave the highest combined sensitivity and specificity, observer reproducibility and fewest indeterminate results. In PE, a mismatch has its base along the pleura and conforms to known sub-segmental and segmental vascular anatomy (Miniati et al., 1996). Applying these principles of interpretation, recent V/P SPECT studies of more than 3000 cases showed a negative predictive value of $97-99 \%$, a sensitivity of $96-99 \%$, and a specificity of $91-98 \%$ for PE diagnosis (Bajc et al., 2008; Leblanc et al., 2007; Lemb \& Pohlabeln, 2001; Miniati et al., 1996; Reinartz et al., 2004). The rate of non-diagnostic findings was $1-3 \%$. V/P SPECT yields ventilation and perfusion images in exactly the same projections, facilitating recognition of mismatch. This is of particular importance in the middle lobe and lingula where mismatch may be overlooked if the lung is not accurately delineated by its ventilation images (Meignan, 2002).

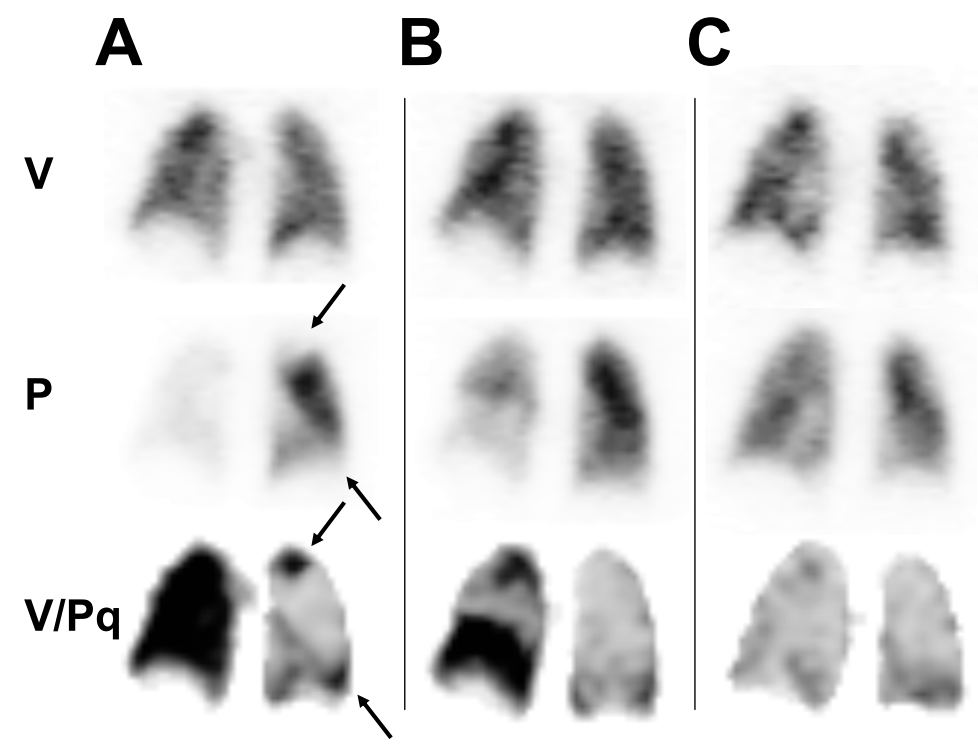

Fig. 4. Patient with massive PE. A) Absent perfusion is seen in the right lung and subsegmental perfusion defects are seen in the left lung (arrows). B) After approximately one week of anticoagulant therapy. C) The day after thrombolysis.

Furthermore, V/P SPECT allows quantification of PE extension which is a prerequisite for individual treatment of PE. As suggested by Olsson et al., the number of segments and subsegments indicating PE are counted and expressed in \% of the total lung parenchyma (Olsson et al., 2006). Moreover, areas with ventilation abnormalities were recognized 
allowing estimation of the degree of total lung malfunction. A segmental reduction or a subsegmental total deficiency of function is attributed 1 point, a segmental total deficiency, 2 points. Each lung comprises according to our charts 9 segments, representing 18 points. Mismatch defects are expressed as mismatch points, which after division by 36 give the fraction of the lung that is embolised. Regions with ventilation or perfusion defects are totalled in order to estimate the reduction in overall lung function.

The study showed that patients with up to $40 \%$ PE could be safely treated at home if ventilation abnormalities engaged less than $20 \%$ of the lung. Since 2004, the Skåne University Hospital, Lund, has successfully treated more than 2000 patients with PE and about $60 \%$ of these patients were treated at home.

\subsection{Diagnosis of pulmonary embolism}

In PE, an embolus blocks the blood flow, causing a perfusion defect, while ventilation remains normal because there is no corresponding blockage in the airway. To characterize the pattern of perfusion defects is crucial. Perfusion defects due to blockage of a pulmonary artery should reflect the branching of pulmonary circulation and its classical segmental anatomy. A segmental defect is wedge shaped with its base on the pleura.

On $V / P$ SPECT images, it is relatively easy to identify segmental and sub-segmental patterns of perfusion defects. Figure 4a shows multiple perfusion defects in acute stage in a female patient with chest pains, who had fainted outside the hospital. Applying quantification on V/P SPECT images, extension of PE was estimated to be ca $60 \%$. The patient was treated first with heparin for a week. However, Follow up showed limited regression (Fig 4b). As brain hemorrhage was excluded, thrombolysis was administered. The following day perfusion was normalized (Fig 4c).

Figure 2 shows sagittal slices of right lung of a patient studied for acute breathlessness. A segmental perfusion defect was well delineated, in perfusion and $V / P_{\text {quotient }}$ images (Fig 2a). Moreover, it was possible to see broncho-constriction in the posterior part of the lung (blue arrow). The extension of perfusion defect was estimated at $10 \%$ and ventilation defect $25 \%$. Three days later nearly complete resolution of the embolus was observed, as well as normalization of the ventilation (Fig 2b).

In planar images, the identification of a solitary segmental perfusion defect within middle lobe and lingula is often impossible or, at best, difficult. With tomographic images these changes are well delineated.

It is important to be aware that mismatch findings not having segmental character do not usually represent PE. Non segmental mismatch means that perfusion defects do not conform to segmental anatomy and are caused by other diseases. This is observed in patients with heart failure (Jögi et al., 2008), pneumonia, mediastinal adenopathy, post radiation therapy etc. Total absence of perfusion in one lung without any other region of mismatch is often caused by pathology other than PE, such as a central tumour or abscess.

\subsection{Follow up}

Follow up is a frequently overlooked aspect of diagnostic strategies although it is essential both for clinical and scientific reasons. The follow up is necessary to assess the effect of treatment, especially to see the effect of anticoagulant therapy (Fig $4 \mathrm{~b}$ ) and in these cases to be able to adjust therapy or, if necessary, continue with thrombolysis (Fig 4c). 
Moreover follow up is important

- To assess the need for prolonged oral anticoagulation beyond 6 months, where there are extensive remnants of $\mathrm{PE}$.

- To allow differentiation between new and old PE, where a recurrence of PE is suspected.

- To explain physical incapacity after PE in case of permanently impaired lung function.

- To evaluate and compare drugs and treatment strategies.

- To identify patients with remaining perfusion defects after treatment as these could be particularly susceptible to developing pulmonary hypertension

For follow up, V/P SPECT is the only suitable method for the following reasons:

- Detection of all emboli requires that the whole lung is examined with a sensitive method.

- The cumulative radiation dose is a central issue when the indication for PE is relative

- $\quad \mathrm{V} / \mathrm{P}$ SPECT is the only method which enables functional impairment to be determined due to increased dead space from non-perfused lung units and increased pulmonary vascular resistance due to a reduced vascular bed.

To be able to study the efficacy of treatment, in individual patients the same method should be used both for diagnosis and follow up. This is a further strong argument in favour of $\mathrm{V} / \mathrm{P}$ SPECT as the primary diagnostic method for PE.
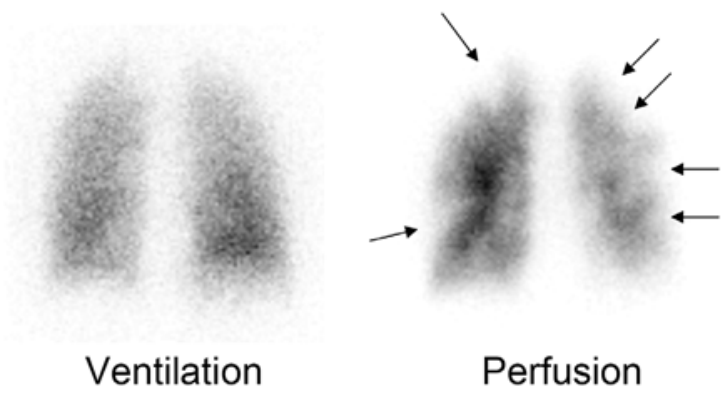

Fig. 5. Patient with chronic PE. Frontal slices. Multiple perfusion defects are seen (arrows).

MDCT was normal.

\subsection{Chronic pulmonary embolism}

Chronic PE is a progressive disease that develops in about $5 \%$ of patients, even after treatment (Begic et al., 2011; Pengo et al., 2004), after an acute episode of PE . However, it often has an insidious onset. It might lead to pulmonary hypertension, right heart failure and arrhythmia, which are frequent causes of death. The value of ventilation/perfusion scintigraphy is well established. It has recently been confirmed in a head to head comparison between MDCT and planar scintigraphy with pulmonary angiography as reference. Among patients with pulmonary hypertension, scintigraphy had a sensitivity of $96-97 \%$ and specificity of $90 \%$, while MDCT had a sensitivity of $51 \%$ (Tunariu et al., 2007). The conclusion was that ventilation/perfusion scintigraphy "has a higher sensitivity than MDCT as well as very good specificity in detecting chronic pulmonary thromboembolic disease as a potentially curable cause of pulmonary hypertension". Scintigraphic features of chronic PE vary. Figure 5 illustrates a case of multiple perfusion defects which are similar to acute PE. MDCT was 
normal. In some patients mismatch without clear segmental or sub-segmental pattern is observed. Peripheral zones of the lung lack perfusion. The centre of the lung is hyperperfused. The lung appears significantly smaller on perfusion images compared to ventilation and the $\mathrm{V} / \mathrm{P}_{\text {quotient }}$ images show mismatch along the lung periphery (Fig 6).

In recent guidelines for the diagnosis and treatment of pulmonary hypertension it is stated that "ventilation/perfusion scan remains the screening method of choice for chronic pulmonary hypertension" (Galie et al., 2009). It was also pointed out that pulmonary venoocclusive disease is a rare but important differential diagnosis.

\section{Right lung. Sagittal.}

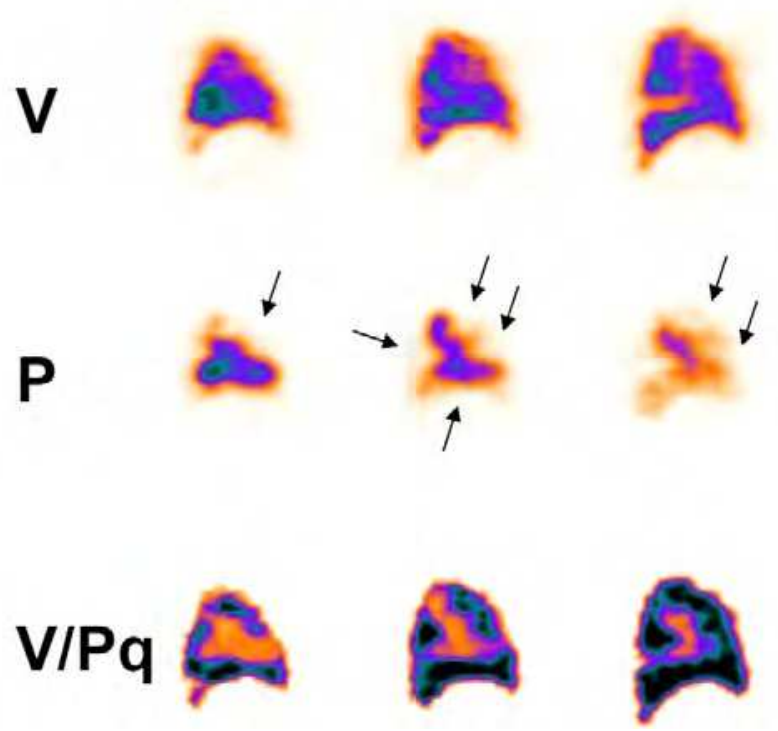

Fig. 6. Patient with pulmonary hypertension caused by chronic PE. Peripheral zones of the lung lack perfusion (arrows). The centre of the lung is hyperperfused $. \mathrm{V} / \mathrm{P}_{\text {quotient }}$ images shows mismatch along the lung periphery.

\section{Sensitivity and specificity of V/P SPECT and other methodological considerations}

In a clinical study, $53 \%$ more mismatch points were identified with V/P SPECT compared to planar technique (Bajc et al., 2004). Similar results have been found by others (Gutte et al., 2010; Reinartz et al., 2001). SPECT eliminates superimposed structures, clarifying segmental and sub-segmental nature of perfusion defects caused by PE.

The value of V/P SPECT is further confirmed in clinical studies (Bajc et al., 2008; Gutte et al., 2009; Leblanc et al., 2007; Lemb \& Pohlabeln, 2001). V/P SPECT is today the recommended method for clinical diagnosis, follow up and research (Bajc et al., 2009b).

Powell reported that sensitivity and specificity of CT for central PE are about $90 \%$ (Powell \& Muller, 2003). Perrier et al. found in a broad clinical material that CT had a sensitivity of $70 \%$ for PE and a specificity of 91 \% (Perrier et al., 2001). They concluded: "clinical CT should not 
be used alone for suspected PE but could replace angiography in combined strategies that include ultrasonography and lung scanning". Likewise, van Strijen et al. found in a multicentre prospective study that sensitivity of CT was $69 \%$ while specificity was $84 \%$ and "concluded that the overall sensitivity of spiral CT is too low to endorse its use as the sole test to exclude PE" and that "this holds true even if one limits the discussion to patients with larger PE in segmental or larger pulmonary artery branches" (Van Strijen et al., 2005). Our experience supports this view. Also, CT as a second procedure following scintigraphy has limited value (van Strijen et al., 2003). Multislice CT seems to improve resolution but sensitivity for small PE appears not to be improved (Stein et al., 2006).

A problem associated with limited sensitivity of CT and incomplete coverage of the total lung is that the degree of embolism and lung function deficiency cannot be quantified. Quantification is important for treatment selection.

In spite of excellent diagnostic qualities of V/P SPECT and documented low sensitivity of $\mathrm{CT}$, the latter method is often recommended. A high number of non-diagnostic scintigraphies were reported in the PIOPED study (65\%) (1990). This is still used as an argument against lung scintigraphy. In PIOPED, scintigraphy was performed with inferior technique and inflexible sub-optimal interpretation criteria. Even with planar scintigraphy, a reduction in the number of non-diagnostic reports to $10 \%$ can be achieved with adequate acquisition and a holistic interpretation strategy (Bajc et al., 2002a). With V/P SPECT, this number is further reduced to between 1 and $4 \%$, as found in several studies (Bajc et al., 2008; Leblanc et al., 2007; Lemb \& Pohlabeln, 2001).

Some practitioners hold that sub-segmental emboli are of little importance for otherwise healthy people and may be left untreated and, as a consequence, are prepared to accept less sensitive methods for PE diagnosis. However, small emboli are important because they 1) may be a first and only sign of silent deep venous thrombosis, 2) may precede larger emboli 3 ) if not diagnosed and/or untreated, further episodes may lead to chronic PE and pulmonary hypertension (Fig. 6). 4) form a threat to patients with limited cardio-pulmonary reserve, 5) are clinically essential for quantification, which is necessary to scientifically establish appropriate treatment protocols. Thus, each embolus is relevant, irrespective of size. Sub-segmental emboli should not be left untreated without further scientific evidence.

\section{Selection of therapeutic strategy}

Management of PE was previously confined to in-hospital therapy, using anticoagulation, heparin injections followed by oral anticoagulants for extended periods of time.

About $20 \%$ to $55 \%$ of patients with deep venous thrombosis have concomitant PE, which is usually not diagnosed because symptoms of PE are absent. Outpatient treatment of patients with deep venous thrombosis, which is perceived as a safe routine, implies that many patients with PE are treated at home. Home treatment of patients with diagnosed PE has been suggested (Kovacs et al., 2000) but in order to determine the appropriateness of the treatment on an individual basis, the extension of PE obviously need to be estimated. Whilst patients with limited extension of PE may be treated at home, intermediate cases and patients with co-morbidity may need in-hospital treatment. Those with very extensive PE may require thrombolysis, necessitating inpatient treatment.

Obviously, quantification requires studies of the whole lung with methods allowing identification of large and small emboli. V/P SPECT is the ideal method for this purpose as segmental and sub-segmental emboli can be both detected with a high degree of sensitivity 
and quantified in terms of mismatch points. In a prospective study Olsson et al. studied 102 out-patients with a moderate degree of PE (up to 40\%) (Olsson et al., 2006). After only 5 days, embolism diminished by $44 \%$ on average. There was no tromboembolic mortality in the trial. Later follow up indicated that PE had not recurred in patients showing resolution after 5 days. Since 2004, more than 1000 patients, of Skåne University Hospital, Lund, with up to $50 \%$ extension of PE have been treated as out-patients. After this positive experience, out-patient treatment is now perceived as a safe routine in patients who have been selected on the basis of V/P SPECT and relevant clinical information.

\section{Additional findings}

$\mathrm{PE}$ is a condition known for its non-specific symptoms. Medical imaging, such as $\mathrm{V} / \mathrm{P}$ SPECT, is therefore necessary to confirm or exclude the diagnosis among patients with suspected PE. The majority of patients that are examined with V/P SPECT, due to the initial assumption of PE, will not have PE. It is therefore important that any alternative diagnoses, which could explain the patients' symptoms, are identified and provided to the referring physician. Possible alternative diagnoses include pneumonia, heart failure, pleural fluid, malignancy and chronic obstructive pulmonary disease (COPD) (Richman et al., 2004). Another important aspect is that these conditions sometimes coexist and that they also elevate the risk of PE (Elliott et al., 2000). V/P SPECT can be employed to identify other diagnoses than PE.

\subsection{Chronic obstructive pulmonary disease}

COPD is a major cause of both morbidity and mortality globally (Mannino et al., 2006). It is one of the few diseases that continues to rise in numbers in many countries. COPD is an inflammatory disease characterized by airflow limitation that is not fully reversible (Celli \& MacNee, 2004). The airflow limitation is caused by a combination of airway obstruction and parenchymal destruction (emphysema). The pulmonary changes in COPD lead to inhomogeneous regional ventilation. V/P SPECT is sufficiently sensitive as a method to identify the functional changes in COPD. In comparison with DTPA aerosols, Technegas penetrates the lung periphery better (Jögi et al., 2010), which is especially important in COPD (exemplified in Fig 3). Technegas ventilation imaging has been shown to visualise the early changes of COPD before they can be observed with high resolution CT (HRCT) (Yokoe et al., 2006). As COPD initially affects the airways, the ventilation defects are commonly more prominent than those of perfusion. Perfusion within the lungs also becomes abnormal as the lungs attempt to adapt the regional blood flow to ventilation to preserve an efficient gas exchange. Often this adaptation is incomplete and perfused but non-ventilated areas (low V/P ratio) occur, i.e. reverse mismatch (Gottschalk et al., 1993). With progressive disease, concurrent destruction of airways and blood vessels takes place and matched defects with absence of both ventilation and perfusion are seen. Vascular remodeling in COPD may lead to regions with elevated V/P ratios. Garg et al. (1983) found that the degree of abnormality on aerosol ventilation images significantly correlated to pulmonary function tests. In a recent paper, which evaluated the role of V/P SPECT in patients with COPD, it was shown that V/P SPECT correlated significantly both to traditional lung function tests as well as the extent of emphysema as measured with HRCT (Jögi et al., 2011). It was also shown that V/P SPECT could be used to characterize the severity of COPD. Pulmonary embolism and heart failure are common comorbidities with overlapping symptoms that 
complicate the manifestation of COPD. The prevalence of PE in patients hospitalized with exacerbation has been reported to be as high as $25 \%$ but is generally under-diagnosed (Mispelaere et al., 2002; Tillie-Leblond et al., 2006). In patients with stable COPD, PE accounts for $10 \%$ of deaths (Schonhofer \& Kohler, 1998). The prevalence of heart failure among patients with stable COPD has been reported to be about $20 \%$ and even higher in patients with exacerbation (Rizkallah et al., 2009; Rutten et al., 2006). The previous finding of the PIOPED study that V/P scintigraphy cannot be used to diagnose PE among patients with pathology of ventilation is an outdated belief (1990). Figure 7 shows extensive PE in a patient with COPD. This heavy smoker had a short history of progressive dyspnea. $\mathrm{V} / \mathrm{P}$ SPECT showed uneven ventilation and perfusion with a pattern that is typical for COPD. Moreover, extensive perfusion defects were seen in areas with preserved ventilation. $V / P$ quotient images could be used to quantify the extent of PE to approximately $70 \%$ of the lung parenchyma.

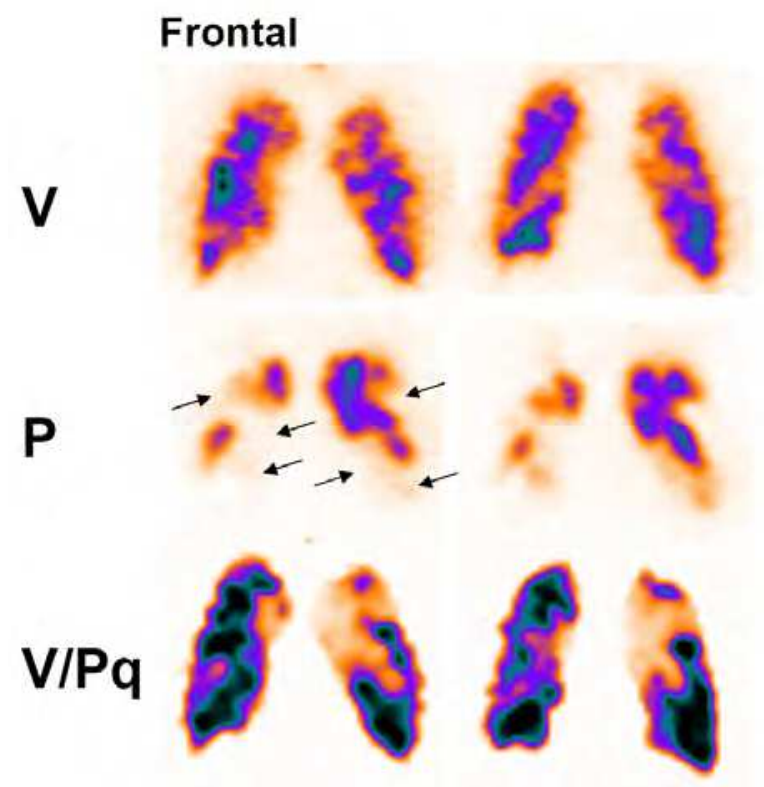

Fig. 7. Patient with severe COPD and chronic PE. Frontal slices; ventilation is very uneven in the whole lung. In addition, multiple perfusion defects are seen in ventilated areas.

Mismatches are highlighted in $\mathrm{V} / \mathrm{P}_{\text {quotient }}$ images.

\subsection{Heart failure}

Left heart failure is a complex clinical syndrome that can result from any cardiac disorder that affects the ability of the left ventricle to function as a pump. When the left chamber is unable to meet the functional demands of the body, symptoms such as dyspnea and fatigue, as well as signs of pulmonary fluid retention appear. In patients with heart failure the risk of developing venous thromboembolic disease, including PE, is elevated (Anderson \& Spencer, 2003). As early as the 1960s, Friedman and Braunwald, West and others demonstrated that patients with mitral valve disease and left heart failure showed an inversion or 
"cephalization" of the normal dependent distribution of blood flow (Friedman \& Braunwald, 1966; West et al., 1964). The inverted distribution of blood flow to nondependent lung zones seen in heart failure has been correlated to elevated pulmonary venous pressure, interstitial oedema, alveolar space flooding and, when longstanding, perivascular fibrosis (Mohsenifar et al., 1989; Pistolesi et al., 1988). Ventilation is not affected to the same degree as perfusion and mismatch in the dependent lung is therefore common. This mismatch, however, is not of a segmental character. V/P SPECT can therefore identify patients with congestive heart failure. In a study of 247 patients examined with V/P SPECT for suspected PE, we found that $15 \%$ of the patients had signs of heart failure, sometimes in combination with PE (Jögi et al., 2008). Figure 8 shows a patient who suffered from increasing breathlessness. V/P SPECT was performed to exclude PE. V/P SPECT in supine position identified redistribution of ventilation and perfusion towards ventral regions (Fig 8a). Perfusion is more affected than ventilation and mismatch is therefore observed. This is, however, non-segmental and the diagnosis was therefore reported as heart failure and not PE. After 12 days of anticongestive treatment, the patient had improved clinically and the follow-up V/P SPECT (Fig 8b) showed complete normalization of both ventilation and perfusion.

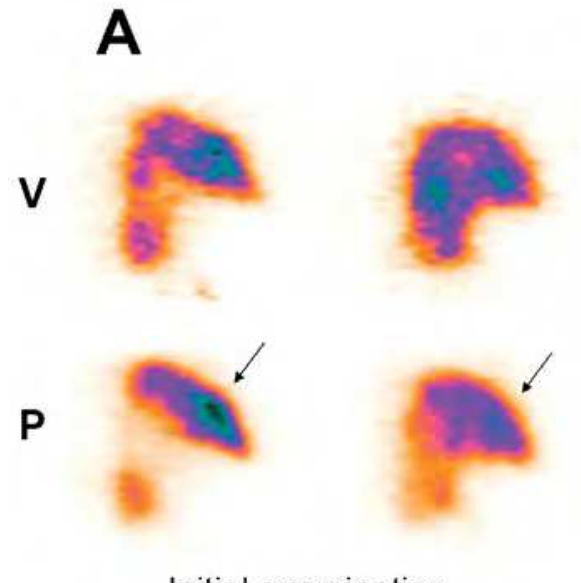

Initial examination

\section{B}

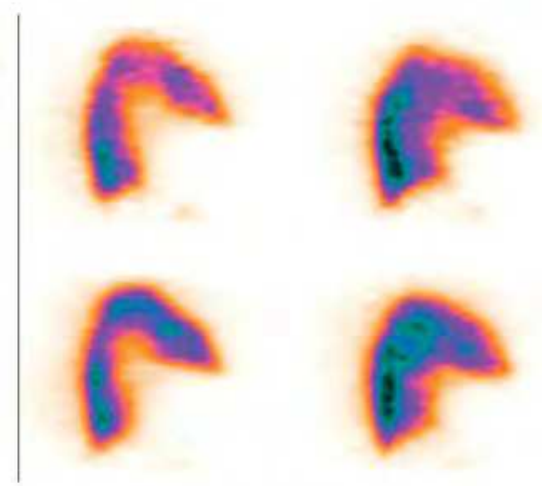

Follow up

Fig. 8. Patient with acute heart failure. A) In the supine patient, perfusion is redistributed to anterior parts of the lung. Ventilation is also affected but not to the same degree, which causes non-segmental perfusion defects. B). Follow up after 12 days of anticongestive treatment

\subsection{Pneumonia}

Pneumonia is an inflammatory condition of the lung parenchyma, especially affecting the alveoli. There are both infectious and non-infectious causes. The pneumonic regions of the lung become non-ventilated although the blood flow is often maintained to some extent. This can cause shunting and lead to hypoxemia. As the ventilation defect often exceeds the perfusion defect, reverse mismatch is a common finding (Carvalho \& Lavender, 1989). In a study by Li et al. (1994) reverse mismatch represented pneumonia in $81 \%$ of the cases. In 
severe cases both ventilation and perfusion are reduced or abolished and matched defects are then frequently found ( $\mathrm{Li}$ et al., 1994). Another sign of pneumonia that often has been described is the "stripe sign" (Sostman \& Gottschalk, 1982, 1992). The "stripe sign" refers to the observation of a stripe of a relatively well preserved perfusion adjacent to the pleural surface, by contrast with the more pronounced perfusion defects within the pneumonic lesion. This distinguishes pneumonia from the segmental perfusion defects found in PE. The enhanced performance of $\mathrm{V} / \mathrm{P}$ SPECT compared to planar imaging has facilitated the detection of the stripe sign (Pace \& Goris, 1998).

Hampson as well as the ICOPER study illustrated that the risk of PE is increased in patients with pneumonia or other coexisting diseases (Elliott et al., 2000; Hampson, 1995). In the ICOPER study, 17\% of 2000 patients with confirmed PE had infiltrates on chest X-ray. Pneumonia has also been shown to be a common finding in patients with autopsy proven PE (Mandelli et al., 1997).

\section{Radiation exposure}

The doses of $30 \mathrm{MBq}$ and $120 \mathrm{MBq}$ for ventilation and perfusion, respectively, allows excellent V/P SPECT quality at an effective radiation dose of $1.8 \mathrm{mSv}$ (ICRP, 1998). The absorbed dose to the breasts with V/P SPECT is $0.8 \mathrm{mGy}$ (ICRP, 1998). Estimation of exposure is relatively easy when short-lived radio-isotopes are used in nuclear medicine. In contrast, for X-ray technologies this is much more difficult because of a number of factors, such as differences in equipment and the size of the exposed fields. According to ICRP, the average effective dose for 4-16-detector MDCT is $5.4 \mathrm{mSv}$ (Valentin, 2007). Notably, this information was based on computed rather than measured dose data. Hurwitz et al. reported for a current adult PE protocol with 64-detector MDCT a measured effective dose of $19.9 \pm 1.38$ $\mathrm{mSv}$ (Hurwitz et al., 2007). These authors point out that the actual measured dose is about $50 \%$ higher than the computed dose. The absorbed dose to the breasts was 35-42 mGy. Absorbed radiation dose to the breast for a single-slice CT study was 20-50 mGy and 30-50\% greater with a four-slice CT. In a very recent study, Hurwitz et al. (Hurwitz et al., 2009) studied radiation dose-saving protocols. Phantoms of women were exposed to MDCT protocols with automatic current modulation, lower tube voltage and bismuth shields over the chest. In the case of a medium sized female patient when automatic current modulation was applied at 140 $\mathrm{kVp}$, breast doses were estimated at $62 \mathrm{mGy}$ and this reduced to $33 \mathrm{mGy}$ when bismuth shields were added. At $120 \mathrm{kVp}$ the doses were $44 \mathrm{mGy}$ without shields and $20 \mathrm{mGy}$ with shields. Some limitations of the study were discussed. No phantom with significant subcutaneous fat was studied. The authors were not able to directly assess the effect of increased noise for the diagnosis of PE. Dose-saving protocols are promising.

\section{Conclusions}

The superiority of V/P SPECT over other imaging techniques in the diagnosis of PE has been well demonstrated. The examination is without contraindication and can be performed in $99 \%$ of patients with suspected PE. V/P SPECT makes it possible to quantify the extent of perfusion loss, so that PE treatment can be better adapted to the needs of individual patients. By using $\mathrm{V} / \mathrm{P}$ SPECT, it is also possible to diagnose other cardiopulmonary conditions.

Because of the very low radiation exposure, it is an ideal method for evaluating treatment and follow up. Low radiation doses are particularly important for women in the reproductive period and during pregnancy. 
The above mentioned advantages of V/P SPECT for studying PE lead to the conclusion that it is the only suitable technique both for follow-up in patients with PE as well as for validation of treatment therapies. Moreover, it is an essential tool for research.

\section{References}

Anderson, F.A., Jr., and Spencer, F.A. (2003). Risk factors for venous thromboembolism. Circulation, Vol. 107, No. 23 Suppl 1, (2003), pp. I9-16

Bajc, M., Albrechtsson, U., Olsson, C.G., Olsson, B., and Jonson, B. (2002a). Comparison of ventilation/perfusion scintigraphy and helical CT for diagnosis of pulmonary embolism; strategy using clinical data and ancillary findings. Clin Physiol Funct Imaging, Vol. 22, No. 6, (2002a), pp. 392-397

Bajc, M., Bitzen, U., Olsson, B., Perez de Sa, V., Palmer, J., and Jonson, B. (2002b). Lung ventilation/perfusion SPECT in the artificially embolized pig. J Nucl Med, Vol. 43, No. 5, (2002b), pp. 640-647

Bajc, M., Neilly, J.B., Miniati, M., Schuemichen, C., Meignan, M., and Jonson, B. (2009a). EANM guidelines for ventilation/perfusion scintigraphy : Part 1. Pulmonary imaging with ventilation/perfusion single photon emission tomography. Eur J Nucl Med Mol Imaging, Vol. 36, No. 8, (2009a), pp. 1356-1370

Bajc, M., Neilly, J.B., Miniati, M., Schuemichen, C., Meignan, M., and Jonson, B. (2009b). EANM guidelines for ventilation/perfusion scintigraphy : Part 2. Algorithms and clinical considerations for diagnosis of pulmonary emboli with $\mathrm{V} / \mathrm{P}(\mathrm{SPECT})$ and MDCT. Eur J Nucl Med Mol Imaging, Vol. 36, No. 9, (2009b), pp. 1528-1538

Bajc, M., Olsson, B., Palmer, J., and Jonson, B. (2008). Ventilation/Perfusion SPECT for diagnostics of pulmonary embolism in clinical practice. Journal of internal medicine, Vol. 264, No. 4, (2008), pp. 379-387

Bajc, M., Olsson, C.G., Olsson, B., Palmer, J., and Jonson, B. (2004). Diagnostic evaluation of planar and tomographic ventilation/perfusion lung images in patients with suspected pulmonary emboli. Clin Physiol Funct Imaging, Vol. 24, No. 5, (2004), pp. 249-256

Begic, A., Jögi, J., Hadziredzepovic, A., Kucukalic-Selimovic, E., Begovic-Hadzimuratovic, S., and Bajc, M. (2011). Tomographic ventilation/perfusion lung scintigraphy in the monitoring of the effect of treatment in pulmonary embolism: serial follow-up over a 6-month period. Nucl Med Commun, Vol. 32, No. 6, (2011), pp. 508-514

Carvalho, P., and Lavender, J.P. (1989). The incidence and etiology of the ventilation/perfusion reverse mismatch defect. Clin Nucl Med, Vol. 14, No. 8, (1989), pp. 571-576

Celli, B.R., and MacNee, W. (2004). Standards for the diagnosis and treatment of patients with COPD: a summary of the ATS/ERS position paper. Eur Respir J, Vol. 23, No. 6, (2004), pp. 932-946

Elliott, C.G., Goldhaber, S.Z., Visani, L., and DeRosa, M. (2000). Chest radiographs in acute pulmonary embolism. Results from the International Cooperative Pulmonary Embolism Registry. Chest, Vol. 118, No. 1, (2000), pp. 33-38

Friedman, W.F., and Braunwald, E. (1966). Alterations in regional pulmonary blood flow in mitral valve disease studied by radioisotope scanning. A simple nontraumatic technique for estimation of left atrial pressure. Circulation, Vol. 34, No. 3, (1966), pp. 363-376 
Galie, N., Hoeper, M.M., Humbert, M., Torbicki, A., Vachiery, J.L., Barbera, J.A., Beghetti, M., Corris, P., Gaine, S., Gibbs, J.S., et al. (2009). Guidelines for the diagnosis and treatment of pulmonary hypertension: the Task Force for the Diagnosis and Treatment of Pulmonary Hypertension of the European Society of Cardiology (ESC) and the European Respiratory Society (ERS), endorsed by the International Society of Heart and Lung Transplantation (ISHLT). Eur Heart J, Vol. 30, No. 20, (2009), pp. 2493-2537

Garg, A., Gopinath, P.G., Pande, J.N., and Guleria, J.S. (1983). Role of radio-aerosol and perfusion lung imaging in early detection of chronic obstructive lung disease. Eur J Nucl Med, Vol. 8, No. 4, (1983), pp. 167-171

Giuntini, C. (2001). Ventilation/perfusion scan and dead space in pulmonary embolism: are they useful for the diagnosis? Q J Nucl Med, Vol. 45, No. 4, (2001), pp. 281-286

Gottschalk, A., Sostman, H.D., Coleman, R.E., Juni, J.E., Thrall, J., McKusick, K.A., Froelich, J.W., and Alavi, A. (1993). Ventilation-perfusion scintigraphy in the PIOPED study. Part II. Evaluation of the scintigraphic criteria and interpretations. J Nucl Med, Vol. 34, No. 7, (1993), pp. 1119-1126

Gray, H.W., McKillop, J.H., and Bessent, R.G. (1993). Lung scan reports: interpretation by clinicians. Nucl Med Commun, Vol. 14, No. 11, (1993), pp. 989-994

Gutte, H., Mortensen, J., Jensen, C.V., Johnbeck, C.B., von der Recke, P., Petersen, C.L., Kjaergaard, J., Kristoffersen, U.S., and Kjaer, A. (2009). Detection of Pulmonary Embolism with Combined Ventilation-Perfusion SPECT and Low-Dose CT: Headto-Head Comparison with Multidetector CT Angiography. J Nucl Med, Vol. 50, (2009), pp. 1987-1992

Gutte, H., Mortensen, J., Jensen, C.V., von der Recke, P., Petersen, C.L., Kristoffersen, U.S., and Kjaer, A. (2010). Comparison of V/Q SPECT and planar V/Q lung scintigraphy in diagnosing acute pulmonary embolism. Nucl Med Commun, Vol. 31, No. 1, (2010), pp. $82-86$

Hampson, N.B. (1995). Pulmonary embolism: difficulties in the clinical diagnosis. Semin Respir Infect, Vol. 10, No. 3, (1995), pp. 123-130

Heck, L.L., and Duley, J.W., Jr. (1974). Statistical considerations in lung imaging with 99mTc albumin particles. Radiology, Vol. 113, No. 3, (1974), pp. 675-679

Howarth, D.M., Booker, J.A., and Voutnis, D.D. (2006). Diagnosis of pulmonary embolus using ventilation/perfusion lung scintigraphy: more than 0.5 segment of ventilation/perfusion mismatch is sufficient. Intern Med J, Vol. 36, No. 5, (2006), pp. 281-288

Hurwitz, L.M., Reiman, R.E., Yoshizumi, T.T., Goodman, P.C., Toncheva, G., Nguyen, G., and Lowry, C. (2007). Radiation dose from contemporary cardiothoracic multidetector CT protocols with an anthropomorphic female phantom: implications for cancer induction. Radiology, Vol. 245, No. 3, (2007), pp. 742-750

Hurwitz, L.M., Yoshizumi, T.T., Goodman, P.C., Nelson, R.C., Toncheva, G., Nguyen, G.B., Lowry, C., and Anderson-Evans, C. (2009). Radiation dose savings for adult pulmonary embolus 64-MDCT using bismuth breast shields, lower peak kilovoltage, and automatic tube current modulation. AJR Am J Roentgenol, Vol. 192, No. 1, (2009), pp. 244-253

ICRP (1998). Radiation dose to patients from radiopharmaceuticals (addendum 2 to ICRP publication 53). Ann ICRP, Vol. 28, No. 3, (1998), pp. 1-126 
James, J.M., Lloyd, J.J., Leahy, B.C., Church, S., Hardy, C.C., Shields, R.A., Prescott, M.C., and Testa, H.J. (1992). 99Tcm-Technegas and krypton-81m ventilation scintigraphy: a comparison in known respiratory disease. Br J Radiol, Vol. 65, No. 780, (1992), pp. 1075-1082

Jögi, J., Ekberg, M., Jonson, B., Bozovic, G., and Bajc, M. (2011). Ventilation/perfusion SPECT in chronic obstructive pulmonary disease: an evaluation by reference to symptoms, spirometric lung function and emphysema, as assessed with HRCT. Eur J Nucl Med Mol Imaging, Vol. 38, No. 7, (2011), pp. 1344-1352

Jögi, J., Jonson, B., Ekberg, M., and Bajc, M. (2010). Ventilation-perfusion SPECT with 99mTc-DTPA versus Technegas: a head-to-head study in obstructive and nonobstructive disease. J Nucl Med, Vol. 51, No. 5, (2010), pp. 735-741

Jögi, J., Palmer, J., Jonson, B., and Bajc, M. (2008). Heart failure diagnostics based on ventilation/perfusion single photon emission computed tomography pattern and quantitative perfusion gradients. Nucl Med Commun, Vol. 29, No. 8, (2008), pp. 666673

Kovacs, M.J., Anderson, D., Morrow, B., Gray, L., Touchie, D., and Wells, P.S. (2000). Outpatient treatment of pulmonary embolism with dalteparin. Thromb Haemost, Vol. 83, No. 2, (2000), pp. 209-211

Leblanc, M., Leveillee, F., and Turcotte, E. (2007). Prospective evaluation of the negative predictive value of V/Q SPECT using 99mTc-Technegas. Nucl Med Commun, Vol. 28, No. 8, (2007), pp. 667-672

Lemb, M., and Pohlabeln, H. (2001). Pulmonary thromboembolism: a retrospective study on the examination of 991 patients by ventilation/perfusion SPECT using Technegas. Nuklearmedizin, Vol. 40, No. 6, (2001), pp. 179-186

Li, D.J., Stewart, I., Miles, K.A., and Wraight, E.P. (1994). Scintigraphic appearances in patients with pulmonary infection and lung scintigrams of intermediate or low probability for pulmonary embolism. Clin Nucl Med, Vol. 19, No. 12, (1994), pp. 1091-1093

Magnussen, J.S., Chicco, P., Palmer, A.W., Bush, V., Mackey, D.W., Storey, G., Magee, M., Bautovich, G., and Van der Wall, H. (1999). Single-photon emission tomography of a computerised model of pulmonary embolism. Eur J Nucl Med, Vol. 26, No. 11, (1999), pp. 1430-1438

Mandelli, V., Schmid, C., Zogno, C., and Morpurgo, M. (1997). "False negatives" and "false positives" in acute pulmonary embolism: a clinical-postmortem comparison. Cardiologia, Vol. 42, No. 2, (1997), pp. 205-210

Mannino, D.M., Watt, G., Hole, D., Gillis, C., Hart, C., McConnachie, A., Davey Smith, G., Upton, M., Hawthorne, V., Sin, D.D., et al. (2006). The natural history of chronic obstructive pulmonary disease. Eur Respir J, Vol. 27, No. 3, (2006), pp. 627-643

Meignan, M.A. (2002). Lung ventilation/perfusion SPECT: the right technique for hard times. J Nucl Med, Vol. 43, No. 5, (2002), pp. 648-651

Miniati, M., Pistolesi, M., Marini, C., Di Ricco, G., Formichi, B., Prediletto, R., Allescia, G., Tonelli, L., Sostman, H.D., and Giuntini, C. (1996). Value of perfusion lung scan in the diagnosis of pulmonary embolism: results of the Prospective Investigative Study of Acute Pulmonary Embolism Diagnosis (PISA-PED). Am J Respir Crit Care Med, Vol. 154, No. 5, (1996), pp. 1387-1393 
Mispelaere, D., Glerant, J.C., Audebert, M., Remond, A., Sevestre-Pietri, M.A., and Jounieaux, V. (2002). [Pulmonary embolism and sibilant types of chronic obstructive pulmonary disease decompensations]. Rev Mal Respir, Vol. 19, No. 4, (2002), pp. 415-423

Mohsenifar, Z., Amin, D.K., and Shah, P.K. (1989). Regional distribution of lung perfusion and ventilation in patients with chronic congestive heart failure and its relationship to cardiopulmonary hemodynamics. Am Heart J, Vol. 117, No. 4, (1989), pp. 887-891

Olsson, C.G., Bitzen, U., Olsson, B., Magnusson, P., Carlsson, M.S., Jonson, B., and Bajc, M. (2006). Outpatient tinzaparin therapy in pulmonary embolism quantified with ventilation/perfusion scintigraphy. Med Sci Monit, Vol. 12, No. 2, (2006), pp. PI9-13

Osborne, D.R., Jaszczak, R.J., Greer, K., Roggli, V., Lischko, M., and Coleman, R.E. (1983). Detection of pulmonary emboli in dogs: comparison of single photon emission computed tomography, gamma camera imaging, and angiography. Radiology, Vol. 146, No. 2, (1983), pp. 493-497

Pace, W.M., and Goris, M.L. (1998). Pulmonary SPECT imaging and the stripe sign. J Nucl Med, Vol. 39, No. 4, (1998), pp. 721-723

Palmer, J., Bitzen, U., Jonson, B., and Bajc, M. (2001). Comprehensive ventilation/perfusion SPECT. J Nucl Med, Vol. 42, No. 8, (2001), pp. 1288-1294

Pengo, V., Lensing, A.W., Prins, M.H., Marchiori, A., Davidson, B.L., Tiozzo, F., Albanese, P., Biasiolo, A., Pegoraro, C., Iliceto, S., et al. (2004). Incidence of chronic thromboembolic pulmonary hypertension after pulmonary embolism. $N$ Engl J Med, Vol. 350, No. 22, (2004), pp. 2257-2264

Perrier, A., Howarth, N., Didier, D., Loubeyre, P., Unger, P.F., de Moerloose, P., Slosman, D., Junod, A., and Bounameaux, H. (2001). Performance of helical computed tomography in unselected outpatients with suspected pulmonary embolism. Ann Intern Med, Vol. 135, No. 2, (2001), pp. 88-97

Pistolesi, M., Miniati, M., Bonsignore, M., Andreotti, F., Di Ricco, G., Marini, C., Rindi, M., Biagini, A., Milne, E.N., and Giuntini, C. (1988). Factors affecting regional pulmonary blood flow in chronic ischemic heart disease. J Thorac Imaging, Vol. 3, No. 3, (1988), pp. 65-72

Powell, T., and Muller, N.L. (2003). Imaging of acute pulmonary thromboembolism: should spiral computed tomography replace the ventilation-perfusion scan? Clin Chest Med, Vol. 24, No. 1, (2003), pp. 29-38, v

Reinartz, P., Schirp, U., Zimny, M., Sabri, O., Nowak, B., Schafer, W., Cremerius, U., and Bull, U. (2001). Optimizing ventilation-perfusion lung scintigraphy: parting with planar imaging. Nuklearmedizin, Vol. 40, No. 2, (2001), pp. 38-43

Reinartz, P., Wildberger, J.E., Schaefer, W., Nowak, B., Mahnken, A.H., and Buell, U. (2004). Tomographic imaging in the diagnosis of pulmonary embolism: a comparison between $\mathrm{V} / \mathrm{Q}$ lung scintigraphy in SPECT technique and multislice spiral CT. J Nucl Med, Vol. 45, No. 9, (2004), pp. 1501-1508

Richman, P.B., Courtney, D.M., Friese, J., Matthews, J., Field, A., Petri, R., and Kline, J.A. (2004). Prevalence and significance of nonthromboembolic findings on chest computed tomography angiography performed to rule out pulmonary embolism: a multicenter study of 1,025 emergency department patients. Acad Emerg Med, Vol. 11, No. 6, (2004), pp. 642-647 
Rizkallah, J., Man, S.F., and Sin, D.D. (2009). Prevalence of pulmonary embolism in acute exacerbations of COPD: a systematic review and metaanalysis. Chest, Vol. 135, No. 3, (2009), pp. 786-793

Rutten, F.H., Cramer, M.J., Lammers, J.W., Grobbee, D.E., and Hoes, A.W. (2006). Heart failure and chronic obstructive pulmonary disease: An ignored combination? Eur J Heart Fail, Vol. 8, No. 7, (2006), pp. 706-711

Schonhofer, B., and Kohler, D. (1998). Prevalence of deep-vein thrombosis of the leg in patients with acute exacerbation of chronic obstructive pulmonary disease. Respiration, Vol. 65, No. 3, (1998), pp. 173-177

Sostman, H.D., and Gottschalk, A. (1982). The stripe sign: a new sign for diagnosis of nonembolic defects on pulmonary perfusion scintigraphy. Radiology, Vol. 142, No. 3, (1982), pp. 737-741

Sostman, H.D., and Gottschalk, A. (1992). Prospective validation of the stripe sign in ventilation-perfusion scintigraphy. Radiology, Vol. 184, No. 2, (1992), pp. 455-459

Stein, P.D., Fowler, S.E., Goodman, L.R., Gottschalk, A., Hales, C.A., Hull, R.D., Leeper, K.V., Jr., Popovich, J., Jr., Quinn, D.A., Sos, T.A., et al. (2006). Multidetector computed tomography for acute pulmonary embolism. N Engl J Med, Vol. 354, No. 22, (2006), pp. 2317-2327

The PIOPED Investigators (1990). Value of the ventilation/perfusion scan in acute pulmonary embolism. Results of the prospective investigation of pulmonary embolism diagnosis (PIOPED). . Jama, Vol. 263, No. 20, (1990), pp. 2753-2759

Tillie-Leblond, I., Marquette, C.H., Perez, T., Scherpereel, A., Zanetti, C., Tonnel, A.B., and Remy-Jardin, M. (2006). Pulmonary embolism in patients with unexplained exacerbation of chronic obstructive pulmonary disease: prevalence and risk factors. Ann Intern Med, Vol. 144, No. 6, (2006), pp. 390-396

Tunariu, N., Gibbs, S.J., Win, Z., Gin-Sing, W., Graham, A., Gishen, P., and Al-Nahhas, A. (2007). Ventilation-perfusion scintigraphy is more sensitive than multidetector CTPA in detecting chronic thromboembolic pulmonary disease as a treatable cause of pulmonary hypertension. J Nucl Med, Vol. 48, No. 5, (2007), pp. 680-684

Wagner, H.N., Jr., Sabiston, D.C., Jr., McAfee, J.G., Tow, D., and Stern, H.S. (1964). Diagnosis of Massive Pulmonary Embolism in Man by Radioisotope Scanning. $N$ Engl J Med, Vol. 271, (1964), pp. 377-384

Valentin, J. (2007). Managing patient dose in multi-detector computed tomography(MDCT). ICRP Publication 102. Ann ICRP, Vol. 37, No. 1, (2007), pp. 1-79, iii

van Strijen, M.J., de Monye, W., Kieft, G.J., Pattynama, P.M., Huisman, M.V., Smith, S.J., and Bloem, J.L. (2003). Diagnosis of pulmonary embolism with spiral CT as a second procedure following scintigraphy. Eur Radiol, Vol. 13, No. 7, (2003), pp. 1501-1507

Van Strijen, M.J., De Monye, W., Kieft, G.J., Pattynama, P.M., Prins, M.H., and Huisman, M.V. (2005). Accuracy of single-detector spiral CT in the diagnosis of pulmonary embolism: a prospective multicenter cohort study of consecutive patients with abnormal perfusion scintigraphy. J Thromb Haemost, Vol. 3, No. 1, (2005), pp. 17-25

West, J.B., Dollery, C.T., and Heard, B.E. (1964). Increased Vascular Resistance in the Lower Zone of the Lung Caused by Perivascular Oedema. Lancet, Vol. 284, (1964), pp. 181183 
Yokoe, K., Satoh, K., Yamamoto, Y., Nishiyama, Y., Asakura, H., Haba, R., and Ohkawa, M. (2006). Usefulness of 99mTc-Technegas and 133Xe dynamic SPECT in ventilatory impairment. Nucl Med Commun, Vol. 27, No. 11, (2006), pp. 887-892 


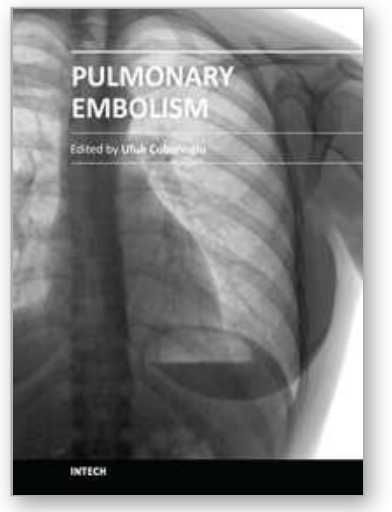

\author{
Pulmonary Embolism \\ Edited by Dr. Ufuk Çobanoğlu
}

ISBN 978-953-51-0233-5

Hard cover, 236 pages

Publisher InTech

Published online 14, March, 2012

Published in print edition March, 2012

Pulmonary embolism is a serious, potentially life-threatening cardiopulmonary disease that occurs due to partial or total obstruction of the pulmonary arterial bed. Recently, new improvement occurred in the diagnosis and treatment of the disease. The aim of this disease is to re-review pulmonary embolism in the light of new developments. In this book, in addition to risk factors causing pulmonary embolus, a guide for systematic approaches to lead the risk stratification for decision making is also presented. In order to provide a maximum length of active life and continuation of functional abilities as the aim of new interventional gerontology, the risk factors causing pulmonary embolus in elderly individuals are evaluated, and the approach to prevention and treatment are defined. The risk of the development of deep vein thrombosis and pulmonary embolism, combined with obesity due to immobility, the disease of this era, irregular and excessive eating, and treatment management are highlighted. Non-thrombotic pulmonary emboli are also covered and an attempt is made to constitute an awareness of this picture that can change the treatment and prognosis of the disease to a considerable extent. In addition to the pathophysiological definition of pulmonary embolus, the priority goal of quick and definitive diagnosis is emphasized, and diagnostic strategies are discussed in the book. A numerical analysis of the vena cava filters, which is a current approach to prevent pulmonary emboli recurrences, is presented in the last chapter.

\title{
How to reference
}

In order to correctly reference this scholarly work, feel free to copy and paste the following:

Marika Bajc and Jonas Jögi (2012). Quantitative Ventilation/Perfusion Tomography: the Foremost Technique for Pulmonary Embolism Diagnosis, Pulmonary Embolism, Dr. Ufuk Çobanoğlu (Ed.), ISBN: 978-953-51-02335, InTech, Available from: http://www.intechopen.com/books/pulmonary-embolism/ventilation-perfusion-spectfirst-hand-method-for-diagnosis-pulmonary-embolism

\section{INTECH}

open science | open minds

\section{InTech Europe}

University Campus STeP Ri

Slavka Krautzeka 83/A

51000 Rijeka, Croatia

Phone: +385 (51) 770447

Fax: +385 (51) 686166

\section{InTech China}

Unit 405, Office Block, Hotel Equatorial Shanghai

No.65, Yan An Road (West), Shanghai, 200040, China 中国上海市延安西路65号上海国际贵都大饭店办公楼405单元

Phone: +86-21-62489820

Fax: +86-21-62489821 
www.intechopen.com 
(C) 2012 The Author(s). Licensee IntechOpen. This is an open access article distributed under the terms of the Creative Commons Attribution 3.0 License, which permits unrestricted use, distribution, and reproduction in any medium, provided the original work is properly cited. 